\title{
Long noncoding RNA DANCR promotes colorectal cancer proliferation and metastasis via miR-577 sponging
}

Yong Wang ${ }^{1}$, Zhi Lu², Ningnin Wang ${ }^{3}$, Jianzhou Feng ${ }^{1}$, Junjie Zhang ${ }^{4}$, Lan Luan ${ }^{4}$, Wei Zhao ${ }^{1}$ and Xiandong Zeng $\mathbb{D}^{5}$

\begin{abstract}
Long non-coding RNAs (IncRNAs) play key roles in various malignant tumors, including colorectal cancer (CRC). Long non-coding RNA differentiation antagonizing non-protein coding RNA (DANCR) is overexpressed in CRC patients, but whether it affects CRC proliferation and metastasis via regulation of heat shock protein 27 (HSP27) remains unclear. In the present study, we found that DANCR was highly expressed and correlated with proliferation and metastasis in CRC. In addition, we demonstrated that DANCR and HSP27 were both targets of microRNA-577 (miR-577) and shared the same binding site. Furthermore, we revealed that DANCR promoted HSP27 expression and its mediation of proliferation/metastasis via miR-577 sponging. Finally, using an in vivo study, we confirmed that overexpression of DANCR promoted CRC tumor growth and liver metastasis. The present study demonstrated the function of DANCR in CRC and might provide a new target in the treatment of CRC.
\end{abstract}

\section{Introduction}

As the third most common cancer, colorectal cancer (CRC) ranks as one of the leading causes of cancer-related death worldwide ${ }^{1}$. It has been reported that approximately more than a half of CRC patients die from distant metastasis, especially liver metastasis ${ }^{2-4}$. Most CRC patients with already existing liver metastasis are not suitable for surgical treatment and therefore have a 5-year survival rate of $<10 \%{ }^{5,6}$. Thus, it is critical to identify new molecules and the underlying mechanisms associated with metastasis development.

Long non-coding RNAs (lncRNAs) are a group of $>200$ nucleotide non-coding RNAs involved in numerous diseases, including CRC. Differentiation antagonizing nonprotein coding RNA (DANCR), located on human

\footnotetext{
Correspondence: Xiandong Zeng (ZXD_landy1116@163.com)

${ }^{1}$ The 4th Department of Orthopedic Surgery, Central Hospital Affiliated to Shenyang Medical College, Shenyang 110024, China

2Department of Nuclear Medicine, The First Affiliated Hospital of Dalian Medical University, Dalian 116011, China

Full list of author information is available at the end of the article

These authors contributed equally: Yong Wang, Zhi Lu.
}

chromosome 4q12, has been reported to act as an oncogene in various malignant tumors. Ma et al. ${ }^{7}$ reported that elevation of DANCR promoted tumor growth and metastasis in hepatocellular carcinoma. Jiang et al. ${ }^{8}$ revealed that DANCR promoted tumor progression and cancer stemness features in osteosarcoma via inhibition of miR-33a-5p. Liu ${ }^{9}$ found that over-expression of DANCR was associated with advanced tumor progression and a poor prognosis in colorectal cancer patients. However, the detailed mechanism concerning how DANCR functions remains unclear.

Among the multiple working mechanisms of lncRNAs, the competitive endogenous RNA (ceRNA) theory was first proposed by Salmena and became prevalent and widely accepted ${ }^{10}$. The ceRNA hypothesis describes crosstalk between lncRNA and RNA transcript that occurs when they share the same miRNA response elements $(\mathrm{MREs})^{11}$. In the present study, we revealed that DANCR and heat shock protein 27 (HSP27), a downstream target of microRNA-577 (miR-577) in CRC, shared binding sites with miR-577. In addition, we found that overexpression of DANCR promoted CRC 


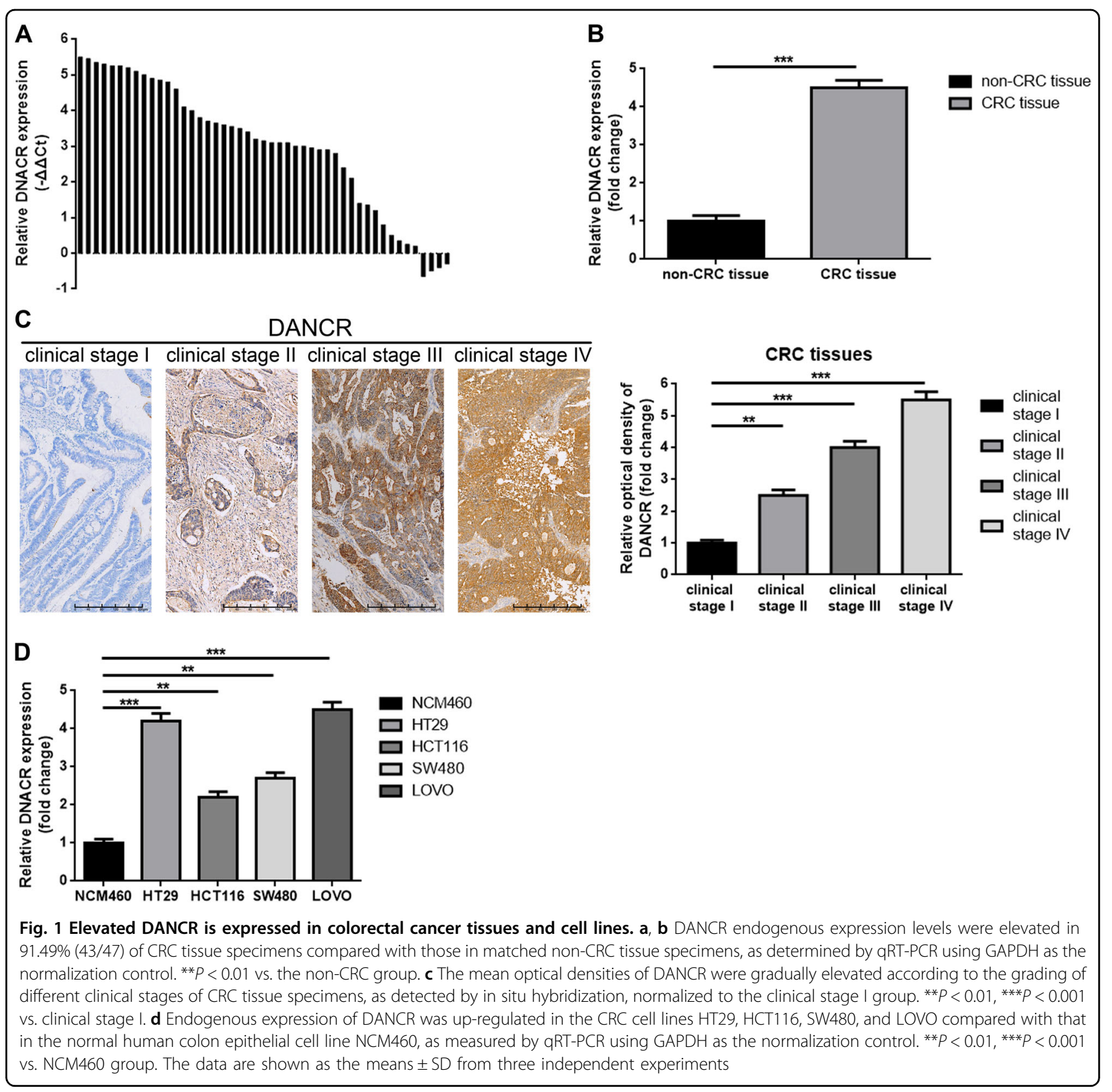

proliferation and metastasis, which were regulated by HSP27 acting as a ceRNA of miR-577.

\section{Materials and methods}

\section{Patient and tissue samples}

In total 47 cases of CRC tissues and matched non-CRC tissues were used in this study and were collected after receiving permission from patients during tumorectomy at the Central Hospital Affiliated to Shenyang Medical College from February 2016 to February 2017. All 47 cases were diagnosed according to a definite pathological diagnosis, and the clinical stage of these patients was determined according to the tumor, node, metastasis (TNM) classification of the International Union Against Cancer (UICC). Written informed consent was obtained from all participants. The Institute Research Medical Ethics Committee of Central Hospital Affiliated to Shenyang Medical College granted approval for this study.

\section{Cell culture}

The human colon cancer cell lines HT29, HCT116, SW480, and LOVO and normal human colon epithelial 
cell line NCM460 were purchased from the American Type Culture Collection and were cultured in RPMI1640 medium (Gibco, El Paso, TX, USA) supplemented with 10 $\%(\mathrm{v} / \mathrm{v})$ fetal bovine serum (FBS, Sigma, St. Louis, MO, USA), $100 \mathrm{IU} / \mathrm{ml}$ penicillin and $100 \mathrm{mg} / \mathrm{ml}$ streptomycin (Baomanbio, Shanghai, China) at $37^{\circ} \mathrm{C}$ in a humidified atmosphere containing $5 \% \mathrm{CO}_{2}$.

Table 1 Correlation of DANCR expression and clinicopathological features in CRC

\begin{tabular}{|c|c|c|c|c|}
\hline \multirow[t]{2}{*}{ Features } & \multirow[t]{2}{*}{ No. of cases } & \multicolumn{2}{|c|}{ DANCR } & \multirow[t]{2}{*}{$p$ value } \\
\hline & & High & Low & \\
\hline Age at diagnosis & & & & $0.571^{*}$ \\
\hline$\leq 50$ & 22 & 11 & 10 & \\
\hline$>50$ & 25 & 11 & 14 & \\
\hline Gender & & & & $0.664^{*}$ \\
\hline Female & 24 & 13 & 11 & \\
\hline Male & 23 & 11 & 12 & \\
\hline Location & & & & $0.671^{\&}$ \\
\hline Colon & 26 & 12 & 14 & \\
\hline Rectal & 21 & 11 & 10 & \\
\hline Clinical stage & & & & $0.043^{\&}$ \\
\hline I & 9 & 3 & 6 & \\
\hline$\|$ & 10 & 3 & 7 & \\
\hline$\|$ & 17 & 12 & 5 & \\
\hline IV & 12 & 9 & 3 & \\
\hline T classification & & & & $0.078^{*}$ \\
\hline $\mathrm{T} 1+\mathrm{T} 2$ & 16 & 6 & 10 & \\
\hline $\mathrm{T} 3+\mathrm{T} 4$ & 31 & 20 & 11 & \\
\hline N classification & & & & $0.016^{\&}$ \\
\hline No & 14 & 4 & 10 & \\
\hline Yes & 33 & 22 & 11 & \\
\hline M classification & & & & $0.003^{\&}$ \\
\hline MO & 15 & 4 & 11 & \\
\hline M1 & 32 & 23 & 9 & \\
\hline \multicolumn{5}{|l|}{ Liver metastasis } \\
\hline No & 19 & 6 & 13 & $0.007^{*}$ \\
\hline Yes & 28 & 20 & 8 & \\
\hline Pathologic differentiation & & & & $0.990^{*}$ \\
\hline Poor & 14 & 8 & 6 & \\
\hline Moderate & 21 & 11 & 10 & \\
\hline Well & 12 & 7 & 5 & \\
\hline
\end{tabular}

P-value obtained from Pearson Chi-Square test ${ }^{*}$ or Fisher's Exact Test ${ }^{\&}$.

\section{Plasmid construction}

DANCR fragments containing miR-577 binding sites were amplified and cloned into pmirGLO vectors (Promega, Madison, WI, USA) to synthesize the wild-type DANCR reporter plasmid pmirGLO-DANCR-wt. The putative binding sites of miR-577 in DANCR were mutated using a QuikChange Site-Directed Mutagenesis kit (Agilent, Santa Clara, CA, USA) to generate the mutant DANCR reporter plasmid pmirGLO-DANCRmut. The pmirGLO-HSP27-wt and pmirGLO-HSP27mut reporter plasmids were constructed using the same method. The above plasmids were used for subsequent luciferase reporter assays. Similarly, DANCR fragments containing miR-577 binding sites were amplified and cloned into the KpnI and XhoI restriction sites (Promega, USA) of the pcDNA3.1 vector to synthesize pcDNA3.1DANCR-wt, and pcDNA3.1-DANCR-mut was also constructed using the QuikChange Site-Directed Mutagenesis kit (Agilent, USA). Four shRNAs against the human DANCR gene (NR_145129.1) (DANCR shRNA) and a non-targeting control sequence (NC shRNA) were ligated into the pLKO.1 plasmid vector (Sigma, USA). These plasmids were used to construct DANCR up-regulation and down-regulation cell models. Meanwhile, the same method was applied to construct the wild-type and mutant HSP27 overexpression plasmids pcDNA3.1HSP27-wt and pcDNA3.1-HSP27-mut, respectively. Oligonucleotides encoding the miR-577 precursor and antimiR-577 precursor were ligated into the pGPH1/GFP/ Neo (pre-miR-577) and pGPU6/GFP/Neo plasmid (antimiR-577) vectors (GenePharma, Shanghai, China), respectively. Plasmids carrying a non-targeting control sequence were used as a negative control (pre-miR-577$\mathrm{NC}$ and anti-miR-577-NC). These plasmids were used to construct the miR-577 up-regulation and downregulation cell models.

\section{Cell transfection}

All of the plasmids used for the construction of the DANCR or miR-577 up-regulation and down-regulation cell models were studied in an up-regulation setting. For transient cell transfection, the CRC cell lines HT29 and LOVO at $60-80 \%$ confluence were prepared for transfection. The plasmids were transfected into HT29 and LOVO cells using Lipofectamine 2000 (Invitrogen, Carlsbad, CA, USA) according to the manufacturer's protocol. For stable cell transfection, cells were selected in culture medium containing $0.4 \mathrm{mg} / \mathrm{ml}$ geneticin (G418; Invitrogen). After 6 weeks, G418-resistant cell clones were established.

\section{Reverse transcription and quantitative real-time PCR}

The procedure was carried out, as previously described $^{12}$. Briefly, total RNA from CRC tissues or 


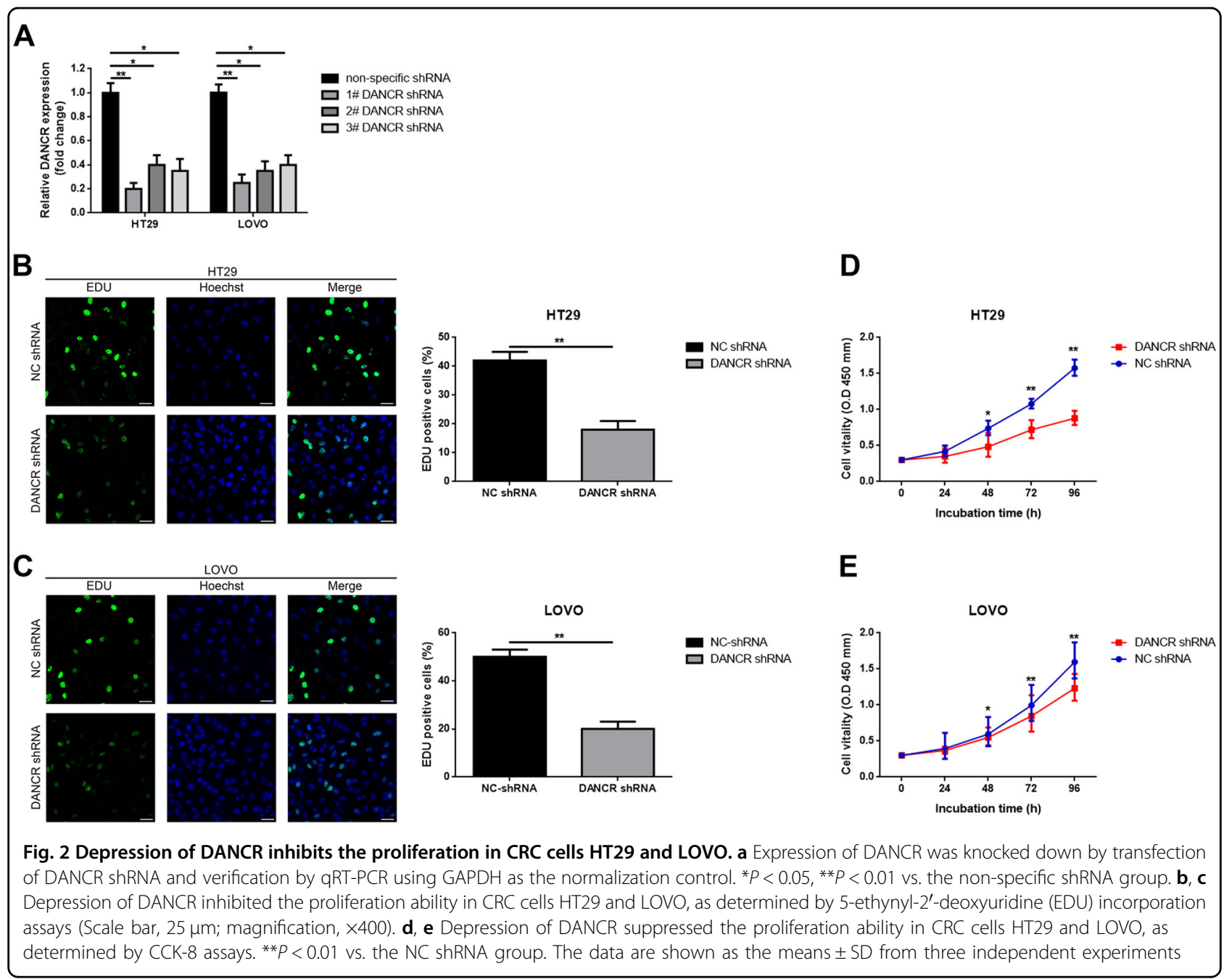

cultured cells was isolated using TRIzol (Invitrogen, USA) according to the manufacturer's protocol. cDNA was synthesized from total RNA using the PrimeScript RT reagent Kit (TaKaRa, Dalian, China). PCR containing SYBR Premix Ex Taq II (TaKaRa, Dalian, China) was carried out according to the manufacturer's instructions.

\section{Western blotting}

The procedures for western blotting were carried out, as previously described ${ }^{13}$. In short, total proteins from CRC tissues or cultured cells were extracted using RIPA lysis buffer (Santa Cruz, USA). Samples were subjected to 10\% SDS-PAGE and transferred to a PVDF membrane. Target proteins were probed using specific antibodies as follows: HSP27 (1:1000; Cell Signaling Technology, USA), LRP6 (1:1000; Cell Signaling Technology, USA) and GAPDH $(0.5 \mu \mathrm{g} / \mathrm{ml}$; Abcam, Cambridge, MA, UK).

\section{Cell proliferation assays}

The 5-ethynyl-20-deoxyuridine (EDU) incorporation assay and Cell Counting Kit-8 (CCK-8) assay were used to assess CRC cell proliferation changes. For the EDU incorporation assay, the procedure was carried out according to the manufacturer's instructions by using EDU detection kits (Ribobio, Guangzhou, China). The CCK- 8 assay was executed, as previously reported ${ }^{14}$. In brief, CRC cells were seeded in 96-well plates $\left(2 \times 10^{3}\right)$ supplemented with complete growth medium, followed by different transfection treatments $24 \mathrm{~h}$ later. At days $1,2,3,4$, and 5 after transfection, $10 \mu \mathrm{l}$ of CCK-8 solution was added to each well, followed by incubation for $2 \mathrm{~h}$. The absorbance was measured at an optical density of $450 \mathrm{~nm}$ using a microplate reader (Bio-Rad, Hercules, CA, USA). Experiments were repeated in triplicate. 


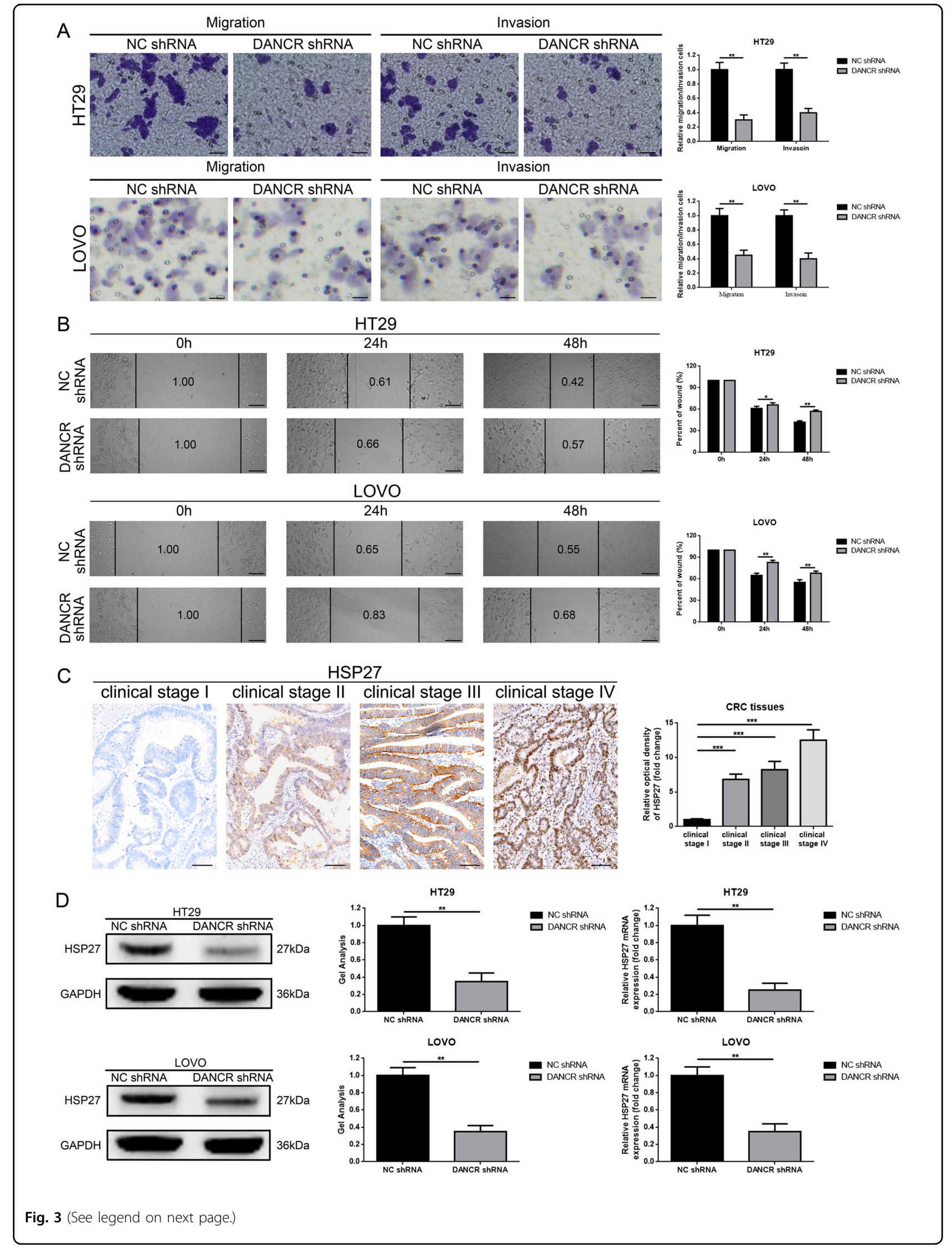


(see figure on previous page)

Fig. 3 Depression of DANCR inhibits metastasis and HSP27 expression in CRC cells HT29 and LOVO. a The metastasis ability of HT29 and LOVO cells was inhibited after the depression of DANCR, as detected by Transwell assays (Scale bar=200 $\mu$ m; magnification, $\times 20$ ). b Depression of DANCR inhibited the metastasis ability of HT29 and LOVO cells, as detected by wound-healing assays (Scale bar=500 $\mu$ m; magnification, $\times 4$ ). $\mathbf{c}$ HSP27 was gradually elevated with progressed staging of CRC as detected by IHC. ${ }^{* *} P<0.001$ vs. the clinical stage I group. $\mathbf{d}$ Depression of DANCR inhibited HSP27 expression in HT29 and LOVO cells, as detected by western blotting and qRT-PCR. ${ }^{* *} P<0.01$ vs. the NC shRNA group. The data are shown as the means \pm SD from three independent experiments

\section{Cell metastasis assays}

The Transwell assay and wound-healing assay were used to detect changes in the metastatic ability of CRC cells. The Transwell assay was performed, as previously reported $^{15}$. After the different interventions, CRC cells were seeded on uncoated and Matrigel-coated upper chambers (BD Bioscience, New Jersey, USA) for migration and invasion assays, respectively. Medium without serum and with 10\% FBS was supplemented into the upper and lower wells, respectively, for an additional $24 \mathrm{~h}$ of incubation. Thereafter, non-migrating or non-invading cells were carefully removed. Next, the filters were fixed in $90 \%$ alcohol, followed by crystal violet staining. Five random fields were counted per chamber using an inverted microscope (Olympus, Tokyo, Japan). For the wound-healing assay, subconfluent monolayers of CRC cells after the different interventions were formed into three parallel lines using a P-200 pipette tip. The detached cells were washed off twice gently, and the medium was then replaced with $1 \%$ FBS complete medium. To visualize wound healing, images were taken at 0 , 24 , and $48 \mathrm{~h}$. The percentage of wound closure (original width-width after cell migration/original width) was calculated. Experiments were repeated in triplicate.

\section{Dual luciferase reporter assay}

The constructed pre-miR-577 or pre-miR-577-NC and reporter plasmids (pmirGLO-HSP27-wt or pmirGLOHSP27-mut and pmirGLO-DANCR-wt or pmirGLODANCR-mut) were co-transfected into cultured CRC cells using Lipofectamine 2000 (Invitrogen, USA) and incubated for $48 \mathrm{~h}$. Next, the Dual-Luciferase Reporter Assay System (Promega, USA) was used to evaluate fluorescence intensity changes according to the manufacturer's protocol.

\section{RNA immunoprecipitation (RIP) assay}

The procedure was carried out as previously described using the Magna RNA-binding protein immunoprecipitation kit (Millipore, Billerica, MA, USA) ${ }^{16}$. In brief, whole-cell lysates were incubated with RIP buffer containing magnetic beads conjugated with a human antiAgo2 antibody or negative-control normal mouse IgG. The samples were incubated with Proteinase $\mathrm{K}$, and then, immunoprecipitated RNA was isolated. The RNA concentration was measured using a spectrophotometer
(Thermo Scientific, Waltham, MA, USA), and the RNA quality was assessed using a bio-analyzer (Agilent, USA). Furthermore, purified RNA was extracted and analyzed by quantitative real-time PCR to demonstrate the presence of the binding targets.

\section{In situ hybridizations assay and immunohistochemistry analysis}

In situ hybridization staining was performed on fresh paraffin sections. Briefly, tissue slides were mixed with 5'-digoxigenin LNA-modified-DANCR (Exiqon A/S, Vedbaek, Denmark) using the IsHyb in situ Hybridization kit (BioChain Institute, Inc., San Francisco, CA, USA) according to the manufacturer's protocol. The immunohistochemistry procedure was performed as previously described $^{17}$.

\section{Animal study}

Male severe combined immunodeficient mice (NODSCID-gamma, 5-6 weeks old) were purchased from the Animal Care and Use Committee of Dalian Medical University Ltd. and were kept under sterile specific pathogenfree conditions. HT29 cells (transfected with pMSCV puroDANCR or pMSCV puro, then selected by G418) with stable overexpression of DANCR $(\mathrm{DANCR}++)$ or the corresponding negative control (DANCR - ) were prepared for further injection. Approximately $1 \times 10^{6}$ prepared HT29 cells in 50\% Matrigel (BD Bioscience, USA) were injected into the subcutaneous and caudal veins of mice. In the subcutaneous group, mice were killed every 4 days for 24 days, tumor growth was measured, and tumor volume was calculated using the formula volume $=1 / 2$ (length $\times$ width ${ }^{2}$ ). After 6 weeks of injection, mice administered a caudal vein injection were dissected to assess liver metastasis. All experimental procedures were carried out in compliance with the guiding principles for the Care and Use of Animals described in the American Journal of Physiology and with the guidelines established by the Institute of Laboratory Animal Sciences, Faculty of Medicine, Kagoshima University. All efforts were made to minimize animal suffering, to reduce the number of animals used, and to utilize possible alternatives to in vivo techniques.

\section{Statistical analysis}

All experiments were repeated in triplicate, and all of the data from three independent experiments were 


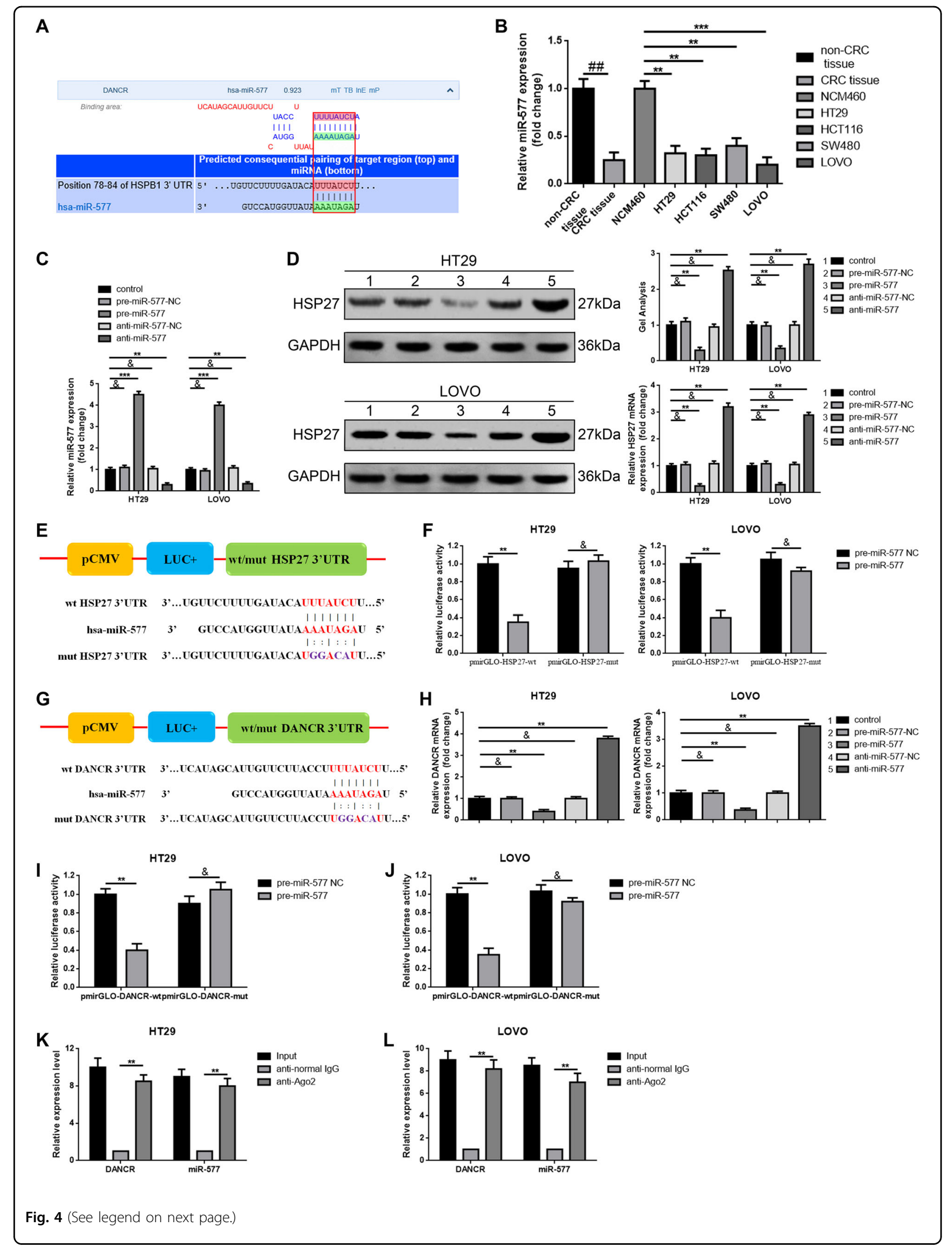




\begin{abstract}
(see figure on previous page)
Fig. 4 DANCR and HSP27 are both targets of miR-577. a DANCR and HSP27 shared the same theoretical miR-577 binding sites, as predicted by targetscan and DIANA-LncBase. b miR-577 was expressed at a lower level in CRC tissues and cell lines than in non-CRC tissues and NCM460, as measured by qRT-PCR. U6 was used as the normalization control. ${ }^{\# \#} P<0.01$ vs. the non-CRC group, ${ }^{* *} P<0.01$ vs. the NCM 460 group. c miR-577 was up- and down-regulated by transfection of pre-miR-577 or anti-miR-577, as detected by qRT-PCR; \&P $>0.05,{ }^{* * *} P<0.001$ vs. control group. $\mathbf{d}$ HSP27 was negatively regulated by miR-577, as determined by qRT-PCR and western blotting; ${ }^{\&} P>0.05,{ }^{* *} P<0.01$ vs. the control group (Nos. $1-5$ represent the control group, pre-miR-577-NC group, pre-miR-577 group, anti-miR-577-NC group and anti-miR-577 group, respectively). e Diagram of the constructed HSP27 reporter plasmid containing the wild-type or mutant miR-577 binding site. f Co-transfection of pre-miR-577 and pmirGLO-HSP27wt led to significant weakening of fluorescence compared with the pre-miR-577 and pmirGLO-HSP27-mut co-transfection group, as determined by luciferase assays. ${ }^{\&} P>0.05,{ }^{*} P<0.01$ vs. the pre-miR-577-NC group. $\mathbf{g}$ Diagram of the constructed DANCR reporter plasmid containing wild-type or mutant miR-577 binding sites. $\mathbf{h}$ DANCR was also negatively regulated by miR-577, as determined by qRT-PCR; ${ }^{*} P>0.05$, ${ }^{* *} P<0.01$ vs. the control group. $\mathbf{i}$, j Weakened fluorescence was present in the pre-miR-577 and pmirGLO-HSP27-wt co-transfection groups, as determined by luciferase assays. ${ }^{\&} P>0.05,{ }^{*} P<0.01$ vs. the pre-miR-577-NC group. $\mathbf{k}$ I I The RIP assay was performed using input from the cell lysate, normal mouse lgG or anti-Ago2. Relative expression levels of DANCR and miR-577 were determined by qRT-PCR. ${ }^{* *} P<0.01$ vs. the anti-normal IgG group. The data are shown as the means \pm SD from three independent experiments
\end{abstract}

expressed as the means \pm SD. GraphPad Prism V5.0 (GraphPad Software, Inc., La Jolla, CA, USA) software and SPSS 19.0 (SPSS, Inc., Chicago, USA) statistical software were used for statistical analysis. $P$-values $<0.05$ were considered statistically significant, while $P$-values $<0.01$ were considered very statistically significant.

\section{Results}

Elevated DANCR is expressed in colorectal cancer tissues and cell lines and is correlated with a poor prognosis in colorectal cancer patients

We detected the DANCR expression level in 47 cases of colorectal cancer tissue specimens and matched noncolorectal cancer tissue specimens using qRT-PCR. As shown in Fig. 1a, b, elevated DANCR was found in $91.49 \%$ (43/47) of colorectal cancer tissue specimens compared with that in non-colorectal cancer tissue specimens ${ }^{* * * *} P$ $<0.001$ ). Simultaneously, DANCR expression was detected in different clinical stages of CRC tissues by in situ hybridization (ISH) analysis. As presented in Fig. 1c, DANCR expression was gradually elevated with the staging of CRC (**P $P<0.01$, $\left.{ }^{* * * *} P<0.001\right)$. Furthermore, we used qRT-PCR to determine the different DANCR expression levels in the CRC cell lines HT29, HCT116, SW480, and LOVO, as well as the normal human colon epithelial cell line NCM460. As shown in Fig. 1d, DANCR expression was remarkably elevated in the CRC cell lines, especially in HT29 and LOVO compared with NCM460; therefore, the HT29 and LOVO cell lines were selected for subsequent cellular experiments $\left({ }^{* *} P<0.01,{ }^{* * *} P<0.001\right)$. Finally, we analyzed the association between the different DANCR expression levels and clinicopathological features of CRC patients using Pearson's chi-squared test or Fisher's exact test. As shown in Table 1, high DANCR expression was closely correlated with clinicopathological features, especially with the clinical stage $(P=0.043)$, nodal $(\mathrm{N})$ classification $(P=0.016)$, metastasis $(\mathrm{M})$ classification $(P=0.003)$, and liver metastasis $(P=0.007)$ (determined by qRT-PCR).

\section{Depression of DANCR inhibits proliferation in CRC cells HT29 and LOVO}

In the previous section, we found that elevated DANCR was closely correlated with the clinical staging. Here, we investigated whether the function of DANCR might work on CRC cell proliferation. We constructed DANCR stable knockdown cell models by transfection of DANCRsilencing plasmids. As shown in Fig. 2a, 1\# DANCR shRNA presented the highest silencing efficiency; therefore, 1\# DANCR shRNA was selected as the silencing plasmid in subsequent RNAi experiments. Next, 5-ethynyl-2'-deoxyuridine (EDU) incorporation assays and CCK-8 assays were used to determine proliferation changes after DANCR knockdown in HT29 and LOVO cells. As presented in Fig. 2b, c (EDU) and 2D-E (CCK-8), depression of DANCR obviously inhibited proliferation in HT29 and LOVO cells $\left({ }^{* *} P<0.01\right)$.

\section{Depression of DANCR inhibits metastasis and decreases HSP27 expression in HT29 and LOVO CRC cells}

In addition, we detected migration/invasion changes in the constructed DANCR knockdown cell models using Transwell assays and wound-healing assays. As the shown in Fig. 3a, b, depression of DANCR led to obvious inhibition of migration/invasion changes in HT29 and LOVO cells $\left({ }^{*} P<0.05,{ }^{* *} P<0.01\right)$.

HSP27, also named HSPB1, has been reported to be a tumor marker, especially regarding cell proliferation and metastasis in colorectal cancer ${ }^{18-22}$. Here, we illustrated that HSP27 was gradually elevated with progressed staging of CRC according to IHC detection (Fig. 3c), indicating that HSP27 is an oncogene, as previously suggested. Furthermore, we detected the influence of DANCR on HSP27 expression. As shown in Fig. 3d, depression of DANCR remarkably decreased HSP27 expression $(* * * 0.01)$.

\section{DANCR and HSP27 are both miR-577 targets}

In the above sections, we demonstrated the expression level and function of DANCR in the colorectal cancer cell 


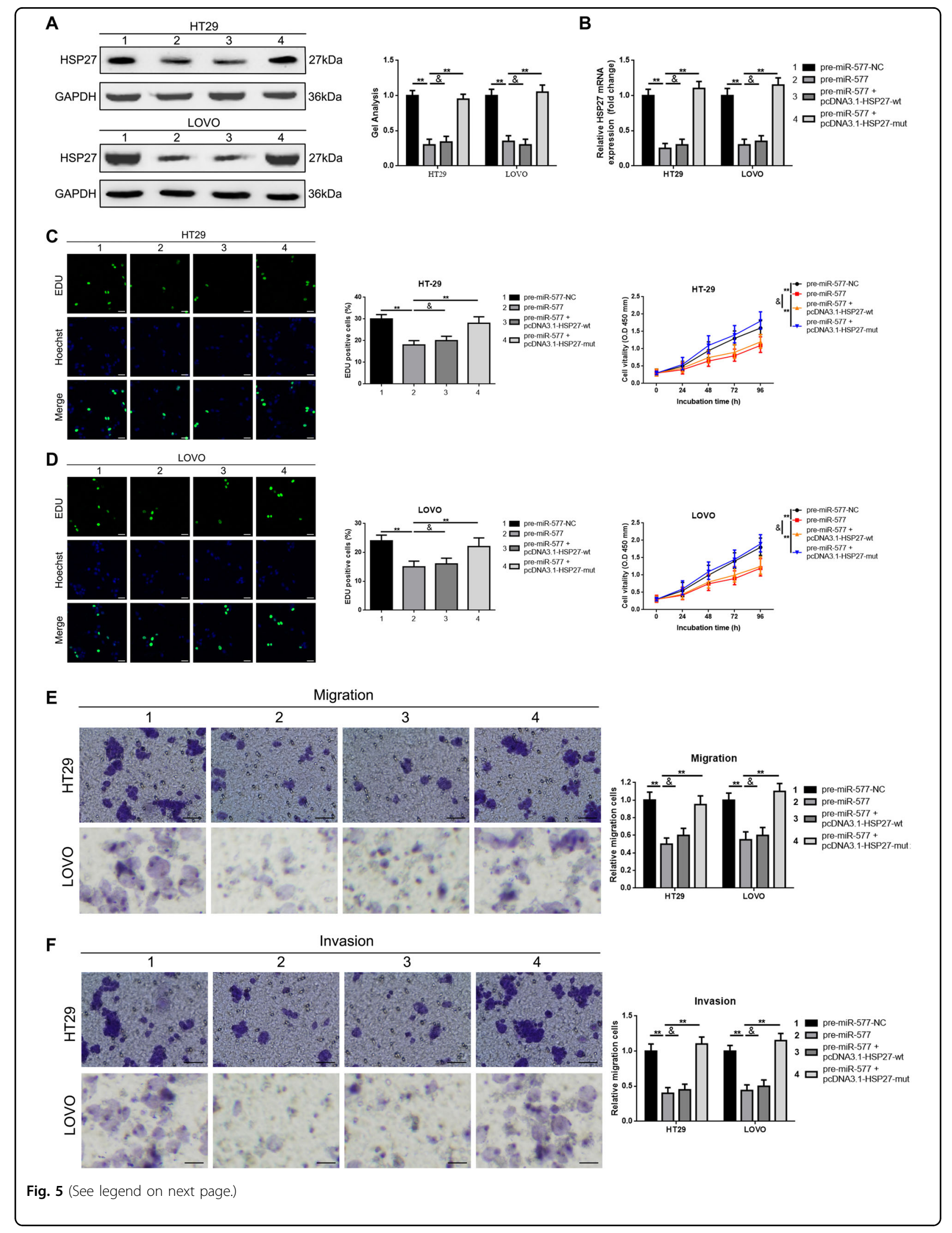


(see figure on previous page)

Fig. 5 MiR-577 suppresses proliferation and metastasis via targeting HSP27 in CRC cells. a, b Up-regulation of miR-577 inhibited HSP27 expression, while the inhibition effect was reversed by pcDNA3.1-HSP27-mut, but not by pcDNA3.1-HSP27-wt, as determined by western blotting (a) and qRT-PCR (b). ${ }^{\&} P>0.05,{ }^{* *} P<0.01$ vs. the pre-miR-577 group. c, d Up-regulation of miR-577 suppressed HT29 (c) and LOVO (d) cell proliferation, while the suppression effect was reversed by pcDNA3.1-HSP27-mut, but not by pcDNA3.1-HSP27-wt, as determined by the EDU and CCK8 assays. \&P $>0.05,{ }^{* *} \mathrm{P}<0.01$ vs. the pre-miR-577 group. e, f Elevation of miR-577 also suppressed HT29 (c) and LOVO (d) cell metastasis, and the suppression effect was reversed by pcDNA3.1-HSP27-mut, but not by pcDNA3.1-HSP27-wt, as determined by the Transwell assay. ${ }^{\&} p>0.05$, ${ }^{* *} P<0.01$ vs. the premiR-577 group (Nos. 1-4 represent the control group, pre-miR-577-NC group, pre-miR-577 group, pre-miR-577 + pcDNA3.1-HSP27-wt group and pre-miR-577 + pcDNA3.1-HSP27-mut group, respectively). The data are shown as the means \pm SD from three independent experiments

lines HT29 and LOVO. Here, we investigated the mechanism underlying the function of DANCR. The competing endogenous RNA (ceRNA) theory is a recent, prevalent theory in the non-coding RNA field. In the previous sections, we confirmed that depression of DANCR inhibited HSP27 expression. Meanwhile, according to a previous study, HSP27 was reported to be an miR-577 target $^{18}$. Thus, we investigated whether a similar ceRNA mechanism exists among DANCR, HSP27 and miR-577. Using the online theoretical prediction programs targetscan and lncbase, we found that DANCR and HSP27 shared the same miR-577 binding sites (at the transcription positions 424-430 in DANCR and 77-83 in HSP27) (Fig. 4a). Next, we confirmed that miR-577 was expressed at a low level in colorectal cancer tissues and cell lines (Fig. $4 \mathrm{~b})\left({ }^{\# \#} P<0.01\right.$, $* P<0.01$, $* * * 0.001)$. Furthermore, we verified that miR577 negatively regulated HSP27 expression by targeting its 3'UTR (Fig. 4c-f). Furthermore, we found that upregulation and down-regulation of miR-577 negatively affected DANCR expression (Fig. 4h) $\left({ }^{* *} P<0.01,{ }^{\&} P>0.05\right)$. Finally, through a luciferase assay and RIP assay, we confirmed that DANCR was a target of miR-577 (Fig. 4g, i-l) $\left({ }^{* *} P<0.01,{ }^{\&} P>0.05\right)$.

\section{MiR-577 suppresses proliferation and metastasis by targeting HSP27 in CRC cells}

In the up-regulation section, we verified that miR-577 targeted HSP27 by binding to its 3'UTR (Fig. 4c-f). Here, we investigated the role of the miR-577/HSP27 axis on CRC cell proliferation and metastasis. Using qRT-PCR and western blotting, we found that pcDNA3.1-HSP27-mut reversed the suppression effect of pre-miR-577 through HSP27 expression, and these findings reconfirmed that miR-577 targeted HSP27 (Fig. 5a, b) $\left({ }^{* * *} P<0.01,{ }^{\circledR} P>0.05\right)$. To further confirm the function of miR-577 in CRC cell proliferation and metastasis, we performed proliferation and metastasis assays. As shown in Fig. 5c, d, elevation of miR-577 (transfection of pre-miR-577) inhibited CRC cell proliferation, while its inhibition effect was reversed by pcDNA3.1-HSP27-mut, but not by pcDNA3.1-HSP27-wt $\left.{ }^{* * *} P<0.01,{ }^{\&} P>0.05\right)$. Moreover, the metastasis assays presented the same tendency as the proliferation assays (Fig. 5e, f) $\quad\left(* * P<0.01,{ }^{\&} P>0.05\right)$. These phenomena confirmed that miR-577 suppressed CRC cell proliferation and metastasis by targeting HSP27.

\section{DANCR promotes proliferation and metastasis via miR-577 sponging in CRC cells}

In this section, elucidated the ceRNA mechanism among DANCR, HSP27 and miR-577. First, we confirmed that the wild-type DANCR overexpression plasmids (transfection of pcDNA3.1-DANCR-wt) promoted expression of HSP27, while the facilitated effect was dismissed when the theoretical binding sites for miR-577 in DANCR were mutated (transfection of pcDNA3.1DANCR-wt). Meanwhile, we found that the elevation of miR-577 (co-transfection of pcDNA3.1-DANCR-wt and pre-miR-577) remarkably alleviated the facilitated effect of pcDNA3.1-DANCR-wt on HSP27 (Fig. 6a) ( $\left.{ }^{\# \#} P<0.01,{ }^{* *} P<0.01,{ }^{\&} P>0.05\right)$. Second, we checked the expression of lipoprotein receptor-related protein 6 (LRP6), another reported downstream mediator of miR $-577^{23}$. In addition, as shown in Fig. 6b, expression of LRP6 presented the same tendency as HSP27 $\left({ }^{\# \#} P<\right.$ $\left.0.01,{ }^{* *} P<0.01,{ }^{\&} P>0.05\right)$. All of the above findings revealed that DANCR promoted HSP27 expression via the miR-577 pathway. Third, we found a reciprocal inhibition effect between DANCR and miR-577 (Fig. 6c, d) $\left({ }^{\# \#} P<0.01, \quad{ }^{* *} P<0.01\right)$. Combined with the aforementioned findings, we concluded that DANCR promoted HSP27 expression via miR-577 sponging. Finally, we performed the proliferation assays and metastasis assays again. As shown in Fig. 6e-h, pcDNA3.1-DANCRwt, but not pcDNA3.1-DANCR-mut, promoted HT29 and LOVO cell proliferation and metastasis, and similar to the expression tendency of HSP27 and LRP6, the facilitated effect of pcDNA3.1-DANCR-wt on proliferation/metastasis was reversed by the elevation of miR-577 $\left({ }^{\# \#} P<0.01, \quad{ }^{* *} P<0.01, \quad{ }^{\&} P>0.05\right)$. In conclusion, our findings convincingly demonstrated that DANCR promoted CRC cell proliferation and metastasis via miR-577 sponging.

\section{Elevation of DANCR promotes tumor growth of CRC in vivo}

To further verify the function of DANCR in CRC tumor growth, HT29 cells with stable overexpression of DANCR $(\mathrm{DANCR}++)$ and the corresponding negative 


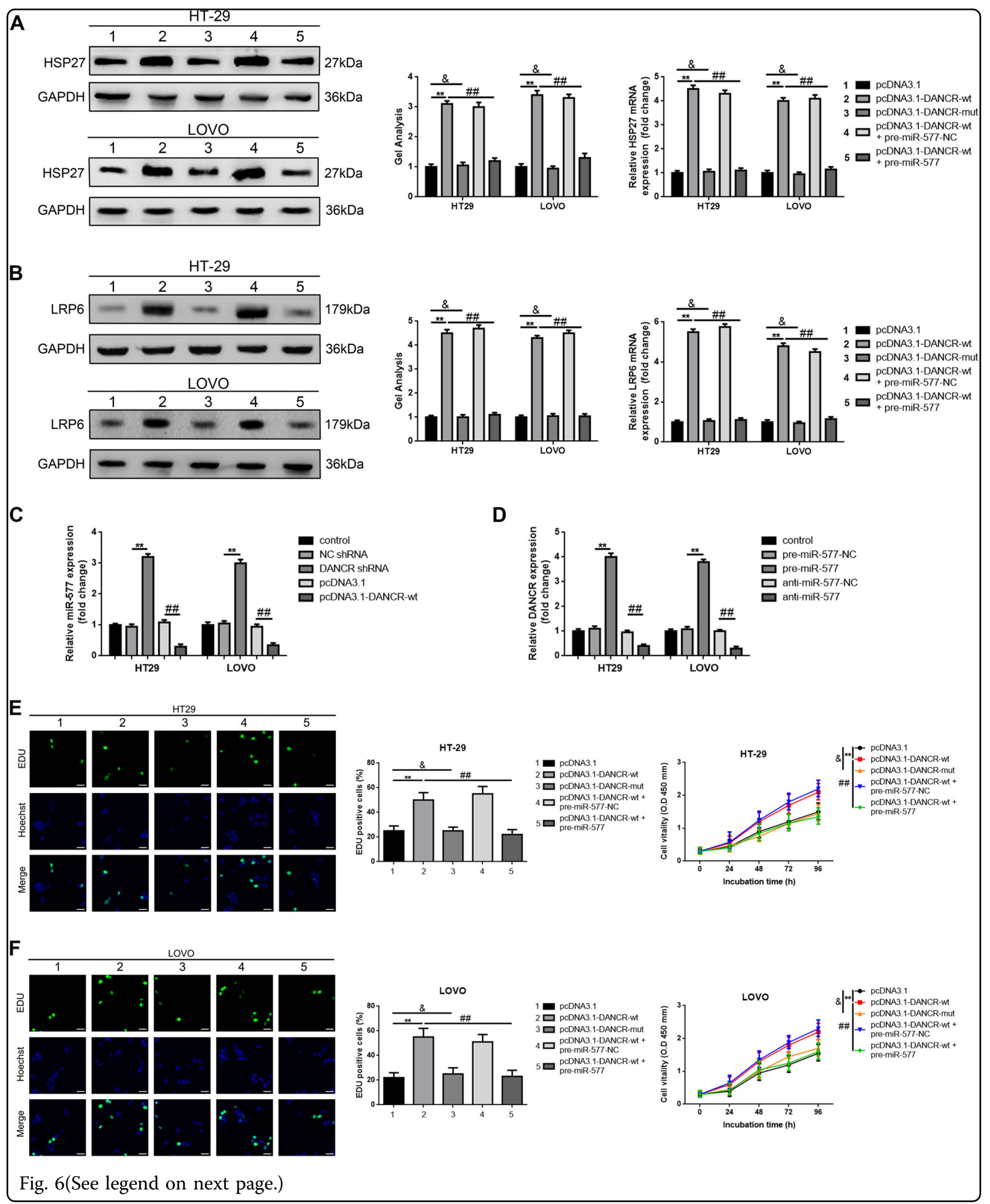




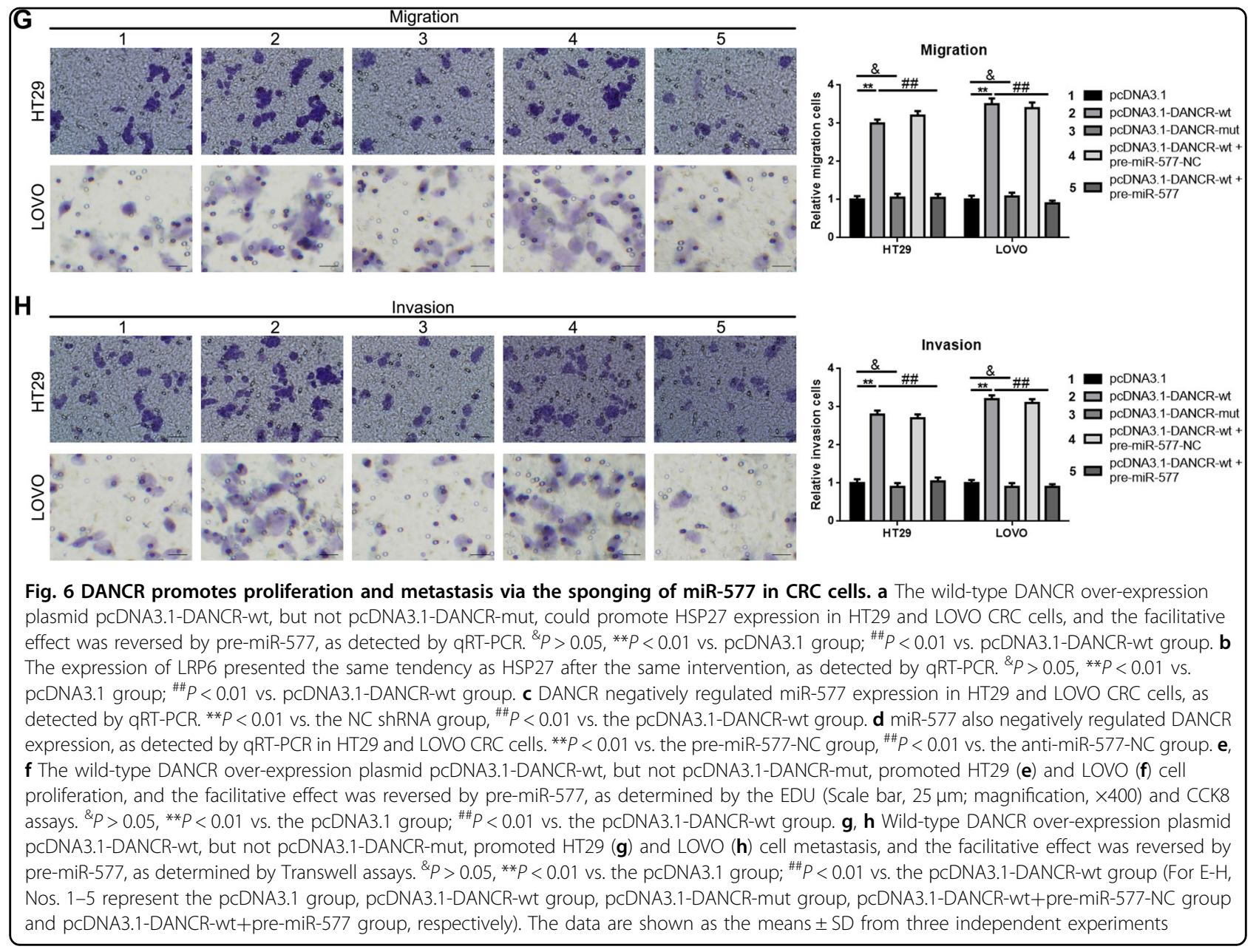

control (DANCR-) were subcutaneously inoculated in nude mice. As the representative pictures shown in Fig. $7 \mathrm{a}-\mathrm{c}$, the volumes of the formatted tumor in the DANCR ++ group were significantly larger than those in the DANCR - group at every detection point (1 week, 2 weeks, 3 weeks, and 4 weeks; $\left.{ }^{* *} P<0.01\right)$. Meanwhile, we detected DANCR and miR-577 expression in the formatted subcutaneous tumors of nude mice in the two groups. As expected, elevated DANCR and depressed miR-577 were presented in the DANCR ++ group compared with those in the DANCR- group (Fig. $7 \mathrm{~d}$, e) ${ }^{* * *} P<$ 0.01). Furthermore, HSP27 expression was determined in the formatted subcutaneous tumors of nude mice in each group according to IHC, qRT-PCR and western blotting. As shown in Fig. 7f, g, HSP27 expression in the DANCR ++ group was higher than that in the DANCRgroup $\left({ }^{* *} P<0.01\right)$.

\section{Elevation of DANCR promotes liver metastasis of CRC in vivo}

Mouse livers after caudal vein injection in the DANCR ++ group and DANCR - group were harvested on the first day of the 7th week. Formatted metastatic nodules were found in $70 \%$ (7 out of 10) of the harvested livers in the DANCR + +group, while the formation rate in the DANCR - group was only 20\% (2 out of 10). As the representative photos and HE staining displayed in Fig. 8a, b, the number of formatted nodules in the DANCR ++ group was significantly higher than that in the DANCR- group $\left({ }^{* *} P<0.01\right)$. Simultaneously, the expression levels of DANCR and miR-577 in the metastatic nodules were detected using qRT-PCR. As the data demonstrates in Fig. 8c, d, expression of DANCR in the DANCR++ group was visibly higher than that in the DANCR- group, but expression of miR-577 presented the opposite trend $(* * P<0.01)$. Finally, HSP27 expression in metastatic liver tumors of nude mice in each group was also determined by IHC, qRT-PCR and western blotting. As shown in Fig. 8e, f, HSP27 expression in the 


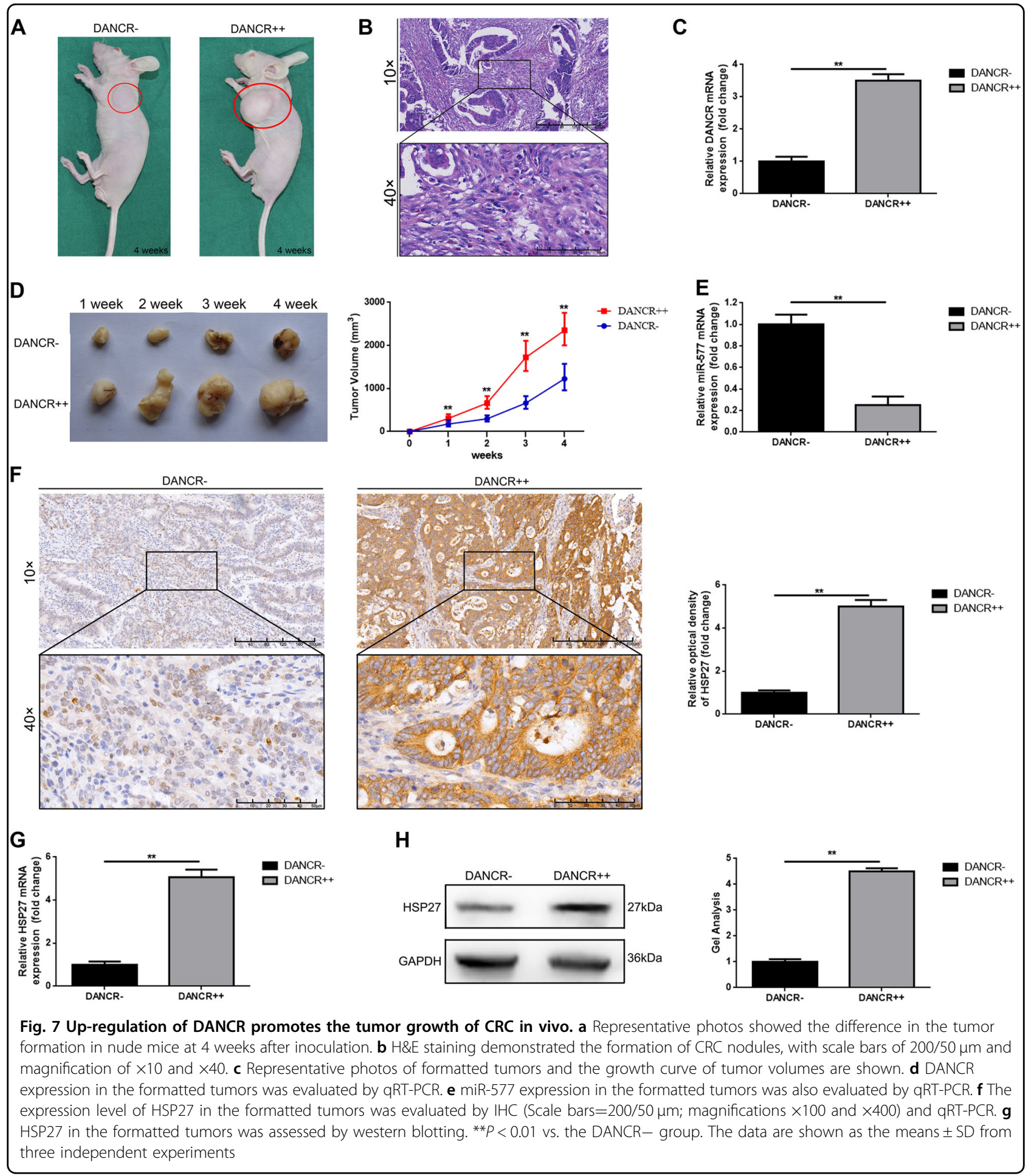

DANCR ++ group was higher than that in the DANCRgroup $(* * P<0.01)$.

\section{Discussion}

lncRNAs are a group of endogenous RNA molecules that contain 200-100,000 nucleotides and are involved in the regulation of gene expression at the epigenetic, transcriptional and post-transcriptional levels in the form of $\mathrm{RNA}^{24}$. IncRNAs exert their functions to regulate gene expression and chromatin structure in the following ways: decoy effect, scaffold effect and post-transcriptional effect $^{25}$. The lncRNA DANCR has been reported to be 


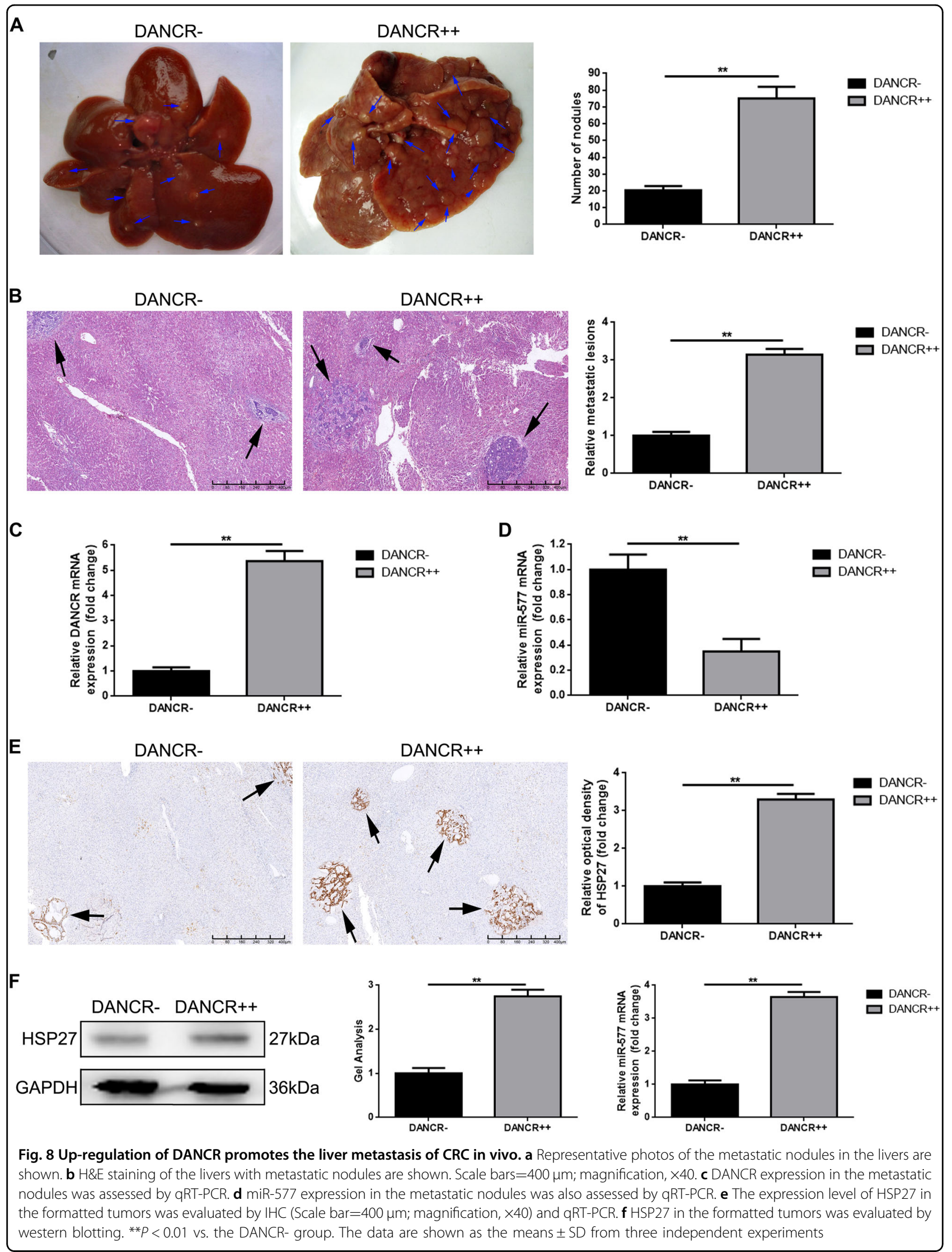




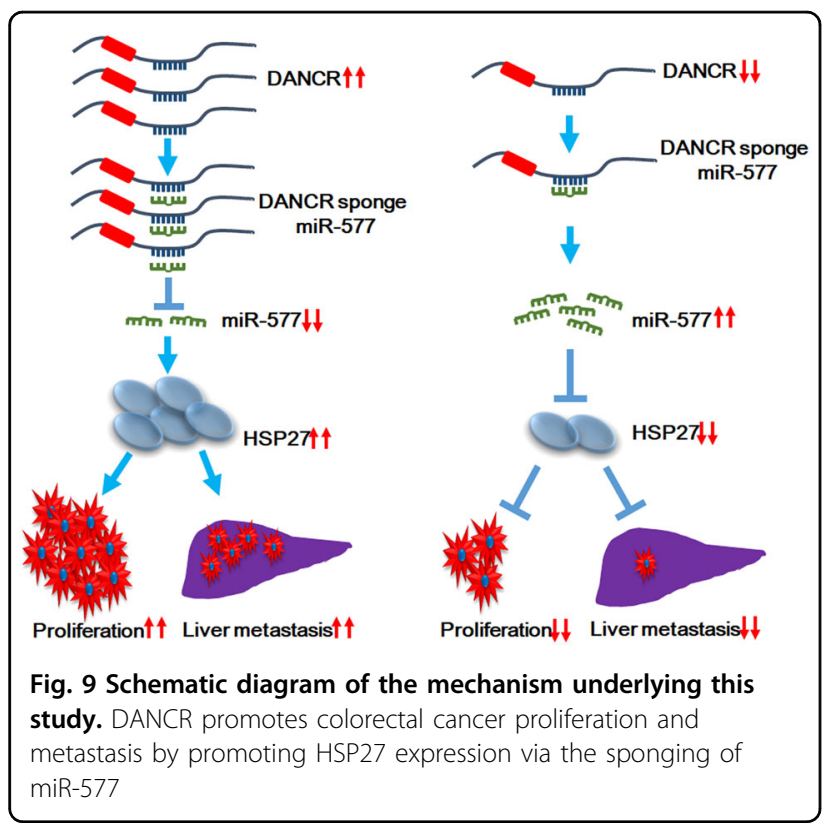

an oncogene in various cancers, including CRC. Jiang reported that DANCR promoted osteosarcoma progression and cancer stemness features via inhibition of miR-33a-5 $\mathrm{p}^{26}$. Jia ${ }^{27}$ found that DANCR promoted prostate cancer invasion via epigenetic silencing of TIMP2/3. Yuan and colleagues ${ }^{28}$ reported that DANCR increased the stemness features of hepatocellular carcinoma by derepression of CTNNB1. In the present study, using clinical specimens and a cellular level of detection, we detected overexpressed DANCR in CRC tissue and cell lines, indicating that DANCR might function as an oncogene in CRC similar to that in other malignant tumors. In addition, the subsequent Chi-squared test indicated that up-regulated DANCR was closely correlated with the clinical stage and occurrence of liver metastasis in CRC patients. Thus, we focused on the function of DANCR in CRC cell proliferation and metastasis. As demonstrated in the functional proliferation and metastasis assays, depression of DANCR inhibited CRC cell proliferation and metastasis. Additionally, these outcomes further confirmed the hypothesis that DANCR is an oncogene in CRC.

Heat shock protein 27 (HSP27) is a member of the small heat shock protein family, and over-expression of HSP27 is associated with promoting drug resistance, aggressive cancers, metastasis, and poor patient outcomes ${ }^{29}$. Currently, there are a number of related reports of HSP27 concerning CRC proliferation and metastasis ${ }^{18,21,30-32}$. Zhang $^{33}$ demonstrated that HSP27 enhanced cell proliferation via activation of activator protein-1. Through detection analysis of 131 human CRC tissue samples, Yuan $^{21}$ suggested that the expression of HSP27 was significantly up-regulated in CRC tissue specimens with lymph node metastasis compared with those without lymph node metastasis and that the down-regulation of HSP27 attenuated the oncogenic effect of miR-145 ${ }^{21}$. HSP27 expression also favors metastasis by influencing epithelial-mesenchymal transition (EMT), in which cells switch from a compact shape to a spindle shape and gain enhanced cell motility ${ }^{34,35}$. In the present study, we also used HSP27 as a breaking point in CRC cell proliferation and metastasis. Through functional inhibition testing, we found that depression of DANCR also suppressed HSP27 expression. Furthermore, through a series of molecular mechanism studies, we revealed the regulatory mechanism of the effect of DANCR on HSP27. Among the working mechanisms of lncRNAs, the competitive endogenous RNA (ceRNA) theory has drawing increasing attention in numerous malignant tumors, including bladder cancer, breast cancer, gastric cancer, hepatocellular cancer and $\mathrm{CRC}^{36-42}$. The ceRNA hypothesis postulates that any RNA transcript that harbors MREs can sequester miRNAs from other targets that share the same MREs, thereby regulating their expression ${ }^{43}$. Through systematic online prediction, we found that DANCR and HSP27 shared the same MREs as miR-577.

It is well known that microRNAs (miRNAs), which are 20-200 nucleotides in length, play key roles in the regulation of various biological processes, including CRC tumorigenesis and metastasis ${ }^{44-50}$. MiR-577, located in human chromosome 4q26, is a member of the miRNA family. As a member of this family, there are few related reports on miR-577 and CRC. Jiang ${ }^{18}$ reported that miR577 was down-regulated in CRC specimens and cell lines and that restoration of miR-577 significantly suppressed proliferation and colony formation and induced G0/G1 cell cycle arrest in CRC cells. In the present study, we found a similar miR-577 expression tendency in tissue and cellular detection as previously reported. It is accepted that miRNAs regulate expression of their target gene by binding to its $3^{\prime} \mathrm{UTR}$. Through a series of miR-577 functional experiments and luciferase assays, we confirmed that both DANCR and HSP27 were the targets of miR-577 because they share the same MREs as miR- 577 . Importantly, we illustrated that miR-577 negatively regulated HSP27-mediated proliferation and invasion in CRC cells.

Recent studies have demonstrated that IncRNAs, which serve as pseudogenes, could inhibit miRNA activity by acting as miRNA "sponges" by sharing some common MREs with miRNAs ${ }^{51}$. In the present study, we found that DANCR and HSP27 shared the same miR-577 MREs, which contain seven basic groups. In addition, we verified that miR-577 targeted DANCR and the HSP27 3'UTR using the same MREs. Furthermore, we confirmed that DANCR and miR-577 inhibited each other's expression reciprocally. Furthermore, using western blotting, qRT- 
PCR and constructed antisense cell behavior experiments, we found that only the wild-type rather than a mutated DANCR overexpression plasmid could promote HSP27 expression and HSP27-mediated CRC cell proliferation/ metastasis. More convincingly, the facilitating effect of DANCR on HSP27 and its mediated cell biological behavior were significantly reversed by pre-miR-577, a constructed miR-577 overexpression plasmid. Hence, as shown in Fig. 9, we conclude that DANCR promotes HSP27 expression and its mediated proliferation and metastasis by acting as a "sponge" or a ceRNA of miR-577 in CRC cells. Finally, we confirmed that overexpression of DANCR promoted CRC tumor growth and liver metastasis in an animal study in vivo.

In conclusion, the present study demonstrated that DANCR was up-regulated in CRC tissue and cell lines. Depression of DANCR inhibited proliferation and metastasis by down-regulating HSP27 via miR-577 sponging in CRC cells in vitro. Elevation of DANCR promoted tumor growth and liver metastasis in constructed nude mouse models. Our findings indicated that DANCR/miR-577/ HSP27axial could be a potential therapeutic target in the molecular targeting treatment of CRC.

\section{Acknowledgements}

This work was supported by the National Natural Science Foundation of China (No. 81502333), PhD Start-up Research Foundation of Liaoning Province (No. 201601225), Natural Science Foundation of Liaoning Province (2015020377), and Scientific Study Project for Institutes of Higher Learning Ministry of Education Liaoning Province (L2015149).

\section{Author details}

${ }^{1} T$ The 4th Department of Orthopedic Surgery, Central Hospital Affiliated to Shenyang Medical College, Shenyang 110024, China. ${ }^{2}$ Department of Nuclear Medicine, The First Affiliated Hospital of Dalian Medical University, Dalian 116011, China. ${ }^{3}$ The 2nd Department of Cardiology, Central Hospital Affiliated to Shenyang Medical College, Shenyang 110024 , China. ${ }^{4}$ Department of Pathology, Central Hospital Affiliated to Shenyang Medical College, Shenyang 110024, China. ${ }^{5}$ Department of Surgical Oncology, Central Hospital Affiliated to Shenyang Medical College, Shenyang 110024, China

\section{Authors' contributions}

Z.X.D. designed the study. Z.W. supervised the study. W.Y., L.Z. and W.N.N. jointly performed the experiments. L.Z. and F.J.Z. contributed to the clinical sample collection and constructed the animal study. Z.J.J. and L.L. performed the pathological analysis. W.Y. and L.Z. analyzed and interpreted the data and drafted the manuscript. All of the authors read and approved the final manuscript.

\section{Conflict of interest}

The authors declare that they have no conflict of interest.

\section{Publisher's note}

Springer Nature remains neutral with regard to jurisdictional claims in published maps and institutional affiliations.

Received: 31 October 2017 Revised: 23 January 2018 Accepted: 14 February 2018.

Published online: 1 May 2018

\section{References}

1. Siegel, R., Desantis, C. \& Jemal, A. Colorectal cancer statistics, 2014. CA Cancer J. Clin. 64, 104-117 (2014).

2. Lan, Y. T. et al. Analysis of the seventh edition of American Joint Committee on colon cancer staging. Int J. Colorectal Dis. 27, 657-663 (2012).

3. Manfredi, S. et al. Epidemiology and management of liver metastases from colorectal cancer. Ann. Surg. 244, 254-259 (2006).

4. $\mathrm{Xu}$, L. et al. MicroRNA-375 suppresses human colorectal cancer metastasis by targeting Frizzled 8. Oncotarget 7, 40644-40656 (2016).

5. Akgul, O., Cetinkaya, E., Ersoz, S. \& Tez, M. Role of surgery in colorectal cancer liver metastases. World J. Gastroenterol. 20, 6113-6122 (2014).

6. Landreau, P. et al. Incidence and survival in late liver metastases of colorectal cancer. J. Gastroenterol. Hepatol. 30, 82-85 (2015).

7. $\mathrm{Ma}, \mathrm{X}$. et al. DANCR acts as a diagnostic biomarker and promotes tumor growth and metastasis in hepatocellular carcinoma. Anticancer Res. 36, 6389-6398 (2016).

8. Jiang, N. et al. InCRNA DANCR promotes tumor progression and cancer stemness features in osteosarcoma by upregulating $A X L$ via miR-33a-5p inhibition. Cancer Lett. 405, 46-55 (2017).

9. Liu, Y., Zhang, M., Liang, L., Li, J. \& Chen, Y. X. Over-expression of IncRNA DANCR is associated with advanced tumor progression and poor prognosis in patients with colorectal cancer. Int J. Clin. Exp. Pathol. 8, 11480-11484 (2015).

10. Salmena, L., Poliseno, L., Tay, Y., Kats, L. \& Pandolfi, P. P. A ceRNA hypothesis: the Rosetta Stone of a hidden RNA language? Cell 146, 353-358 (2011).

11. Song, Y. X. et al. Non-coding RNAs participate in the regulatory network of CLDN4 via ceRNA mediated miRNA evasion. Nat. Commun. 8, 289 (2017).

12. Wang, Y. et al. Long non-coding RNA TUG1 promotes migration and invasion by acting as a ceRNA of miR-335-5p in osteosarcoma cells. Cancer Sci. 108, 859-867 (2017).

13. Kong, J. et al. Long non-coding RNA LINC01133 inhibits epithelialmesenchymal transition and metastasis in colorectal cancer by interacting with SRSF6. Cancer Lett. 380, 476-484 (2016).

14. Wang, Y. et al. Decrease of miR-195 promotes chondrocytes proliferation and maintenance of chondrogenic phenotype via targeting FGF-18 pathway. Int. J. Mol. Sci. 18, 975 (2017).

15. Wang, Y., Zhao, W. \& Fu, Q. miR-335 suppresses migration and invasion by targeting ROCK1 in osteosarcoma cells. Mol. Cell Biochem. 384, 105-111 (2013).

16. Cai, $\mathrm{H}$. et al. Long non-coding RNA taurine upregulated 1 enhances tumorinduced angiogenesis through inhibiting microRNA-299 in human glioblastoma. Oncogene 36, 318-331 (2017).

17. Wang, $Y$. et al. MicroRNA-335 and its target Rock1 synergistically influence tumor progression and prognosis in osteosarcoma. Oncol. Lett. 13, 3057-3065 (2017).

18. Jiang $H$, Ju H, Zhang L, Lu H, Jie K. microRNA-577 suppresses tumor growth and enhances chemosensitivity in colorectal cancer. J. Biochem. Mol Toxicol. 31 https://doi.org/10.1002/jbt.21888 (2017).

19. Liu, W. et al. Identification of HSP27 as a potential tumor marker for colorectal cancer by the two-dimensional polyacrylamide gel electrophoresis. Mol. Biol. Rep. 37, 3207-3216 (2010).

20. Wang, F., Zhang, P., Shi, C., Yang, Y. \& Qin, H. Immunohistochemical detection of HSP27 and hnRNP K as prognostic and predictive biomarkers for colorectal cancer. Med. Oncol. 29, 1780-1788 (2012).

21. Yuan, W. et al. Up-regulation of microRNA-145 associates with lymph node metastasis in colorectal cancer. PLOS ONE 9, e102017 (2014).

22. Zeng, $H$. \& Botnen, J. H. Selenium is critical for cancer-signaling gene expression but not cell proliferation in human colon Caco-2 cells. Biofactors 31, 155-164 (2007).

23. Zhang, W. et al. miR-577 inhibits glioblastoma tumor growth via the Wnt signaling pathway. Mol. Carcinog. 55, 575-585 (2016).

24. Chen, L. L. \& Carmichael, G. G. Long noncoding RNAs in mammalian cells: what, where, and why? Wiley Interdiscip. Rev. RNA 1, 2-21 (2010).

25. Derrien, $\mathrm{T}$. et al. The GENCODE v7 catalog of human long noncoding RNAs: analysis of their gene structure, evolution, and expression. Genome Res. 22, 1775-1789 (2012).

26. Zhang $L$, et al. Long noncoding RNA DANCR regulates miR-1305-Smad 4 axis to promote chondrogenic differentiation of human synovium-derived mesenchymal stem cells. Biosci. Rep. 37, 1-11 (2017). 
27. Jia, J. et al. Long noncoding RNA DANCR promotes invasion of prostate cancer through epigenetically silencing expression of TIMP2/3. Oncotarget 7, 37868-37881 (2016)

28. Yuan, S. X. et al. Long noncoding RNA DANCR increases stemness features of hepatocellular carcinoma by derepression of CTNNB1. Hepatology 63, 499-511 (2016).

29. McConnell, J. R. \& McAlpine, S. R. Heat shock proteins 27, 40, and 70 as combinational and dual therapeutic cancer targets. Bioorg. Med. Chem. Lett. 23, 1923-1928 (2013)

30. Hung, C. S. et al. IGFBP2 plays an important role in heat shock protein 27mediated cancer progression and metastasis. Oncotarget 8, 54978-54992 (2017).

31. Konda, J. D., Olivero, M., Musiani, D., Lamba, S. \& Di Renzo, M. F. Heat-shock protein 27 (HSP27, HSPB1) is synthetic lethal to cells with oncogenic activation of MET, EGFR and BRAF. Mol. Oncol. 11, 599-611 (2017).

32. Zhao, L., Li, Z. G. \& Ding, Y. Q. Expression of HSP27 in colorectal carcinoma and its relationship with lymphatic metastasis. Nan Fang. Yi Ke Da Xue Xue Bao $\mathbf{2 8}$ 41-44 (2008).

33. Zhang, S. et al. Heat shock protein 27 promotes cell proliferation through activator protein-1 in lung cancer. Oncol. Lett. 9, 2572-2576 (2015).

34. Pavan, S. et al. HSP27 is required for invasion and metastasis triggered by hepatocyte growth factor. Int J. Cancer 134, 1289-1299 (2014).

35. Shiota, M. et al. Hsp27 regulates epithelial mesenchymal transition, metastasis, and circulating tumor cells in prostate cancer. Cancer Res. 73, 3109-3119 (2013).

36. Guo, L. L. et al. Competing endogenous RNA networks and gastric cancer. World J. Gastroenterol. 21, 11680-11687 (2015).

37. Huang, M. et al. Comprehensive analysis of differentially expressed profiles of IncRNAs and circRNAs with associated co-expression and ceRNA networks in bladder carcinoma. Oncotarget 7, 47186-47200 (2016).

38. Li, X. et al. STARD13-correlated ceRNA network inhibits EMT and metastasis of breast cancer. Oncotarget 7, 23197-23211 (2016).

39. Liang, W. C. et al. The IncRNA H19 promotes epithelial to mesenchymal transition by functioning as miRNA sponges in colorectal cancer. Oncotarget 6 , 22513-22525 (2015)
40. Wang, $H$. et al. Comprehensive analysis of aberrantly expressed profiles of IncRNAs and miRNAs with associated ceRNA network in muscle-invasive bladder cancer. Oncotarget 7, 86174-86185 (2016).

41. Wu, H. et al. Comprehensive analysis of differentially expressed profiles of IncRNAs and construction of miR-133b mediated ceRNA network in colorectal cancer. Oncotarget 8, 21095-21105 (2017).

42. Zhang, J., Fan, D., Jian, Z., Chen, G. G. \& Lai, P. B. Cancer specific long noncoding rnas show differential expression patterns and competing endogenous RNA potential in hepatocellular carcinoma. PLOS ONE 10, e0141042 (2015).

43. Karreth, F. A. \& Pandolfi, P. P. ceRNA cross-talk in cancer: when ce-bling rivalries go awry. Cancer Discov. 3, 1113-1121 (2013).

44. Bu, P. et al. miR-1269 promotes metastasis and forms a positive feedback loop with TGF-beta. Nat. Commun. 6, 6879 (2015).

45. Li, W. et al. miRNA-99b-5p suppresses liver metastasis of colorectal cancer by down-regulating mTOR. Oncotarget 6, 24448-24462 (2015).

46. Li, X., Nie, J., Mei, Q. \& Han, W. D. MicroRNAs: novel immunotherapeutic targets in colorectal carcinoma. World J. Gastroenterol. 22, 5317-5331 (2016).

47. Mohammadi, A., Mansoori, B. \& Baradaran, B. The role of microRNAs in colorectal cancer. Biomed. Pharmacother. 84, 705-713 (2016).

48. Muhammad, S. et al. MicroRNAs in colorectal cancer: role in metastasis and clinical perspectives. World J. Gastroenterol. 20, 17011-17019 (2014).

49. Yin, Y. et al. Systematic analysis of key miRNAs and related signaling pathways in colorectal tumorigenesis. Gene 578, 177-184 (2016).

50. Zhang, L. \& Fan, X. M. The pathological role of microRNAs and inflammation in colon carcinogenesis. Clin. Res. Hepatol. Gastroenterol. 39, 174-179 (2015).

51. Sen, R., Ghosal, S., Das, S., Balti, S. \& Chakrabarti, J. Competing endogenous RNA: the key to posttranscriptional regulation. Sci. World J. 2014, 896206 (2014). 